\title{
Virulência de fungos entomopatogênicos a Enneothrips flavens (Thysanoptera: Thripidae)
}

\section{Submetido - 28 jul. 2020 \\ Aprovado - 20 set. 2020 \\ Publicado - \\ out. 2020 \\ http://dx.doi.org/10.17648/sas.v1i2.94}

\begin{abstract}
Agrônomo e Mestre em Agronomia (Fitossanidade) - Universidac
\end{abstract} luc2090@gmail.com. \section{Agrônomo e Mestre em Agronomia - Universidade Federal de Uberlândia (UFU),} gabriel.f.rezende@hotmail.com.

Cintia de Melo Gomes (iD Agrônoma - Universidade Federal de Uberlândia (UFU), cintiamelogomes@gmail.com.

Agrônomo e Mestre em Agronomia (Entomologia Agrícola) - Universidade Estadual Paulista (UNESPFCAV), ricardolima_01@hotmail.com.

Agrônomo e Doutor em Entomologia - Universidade de São Paulo (USP-ESALQ), rapolanc@yahoo.com.br.

\section{RESUMO}

A importância do Brasil como produtor de amendoim tem crescido nos últimos anos principalmente na produção de óleo vegetal e de subprodutos. Embora os produtores tenham adotado sistemas de produção mais eficientes, as perdas causadas por pragas têm sido um grande problema. Enneothrips flavens têm sido a principal praga do amendoim no Brasil. 0 controle de $E$. flavens tem sido realizado utilizando-se produtos químicos sintéticos. Objetivou-se com este trabalho avaliar a patogenicidade dos fungos Beauveria bassiana $e$ Metarhizium anisopliae de produtos comerciais e da coleção da UNESP/FCAV sobre ninfas de E. flavens. Esporos destes fungos, na concentração de $10^{8} \mathrm{~mL}^{-1}$ foram aplicados sobre 0 folíolo de plantas de amendoim contendo 10 ninfas de $E$. flavens. A mortalidade das ninfas foi avaliada após 3, 5 e 7 dias de observação. M. anisopliae (ARSEF 3293) e a cepa do produto comercial (Metarril@) apresentaram valores de mortalidade de $34 \%$ e $32 \%$, respectivamente. $A$ cepa B. bassiana do produto comercial (Boveril(B) e a cepa B. bassiana (ARSEF 3288) apresentaram valores de mortalidade de $76 \%$ e $44 \%$, respectivamente. Os dados obtidos neste trabalho sugerem que os isolados ARSEF 3293 e ARSEF 3288, assim como as cepas comerciais, têm potencial para serem usados como agente de controle biológico de $E$. flavens.

Palavras-chave: Amendoim; Controle biológico; Tripes; Beauveria bassiana, Metarhizium anisopliae.

\section{Virulence of entomopathogenic fungi to Enneothrips flavens (Thysanoptera: Thripidae)}

\begin{abstract}
Brazil's importance as a peanut producer has grown in recent years mainly in the production of vegetable oil and by-products. Although producers have adopted more efficient production systems, the losses caused by pests have been a major problem. Enneothrips flavens have
\end{abstract}


been the main pest of peanuts in Brazil. The control of E. flavens has been carried out using synthetic chemicals. The objective of this work was to evaluate the pathogenicity of fungi Beauveria bassiana and Metarhizium anisopliae from commercial products and from the UNESP I FCAV collection on E. flavens nymphs. Spores from these fungi, at a concentration of $10^{8} \mathrm{~mL}^{-1}$, were applied to the peanut plant leaflet containing $10 \mathrm{E}$. flavens nymphs. Nymph mortality was assessed after 3, 5 and 7 days of observation. M. anisopliae (ARSEF 3293) and the commercial product strain (Metarril $(B)$ showed mortality values of $34 \%$ and $32 \%$, respectively. The $B$. bassiana strain of the commercial product (Boveril $(B)$ and the B. bassiana strain (ARSEF 3288) presented mortality values of $76 \%$ and $44 \%$, respectively. The data obtained in this work suggest that the isolates ARSEF 3293 and ARSEF 3288, as well as commercial strains, have the potential to be used as a biological control agent for $E$. flavens.

Keywords: Peanut; Biological control; Thrips; Beauveria bassiana; Metarhizium anisopliae.

\title{
Virulencia de hongos entomopatógenos a Enneothrips flavens (Thysanoptera: Thripidae)
}

\begin{abstract}
RESUMEN
La importancia de Brasil como productor de maní ha crecido en los últimos años principalmente en la producción de aceite vegetal y subproductos. Aunque los productores han adoptado sistemas de producción más eficientes, las pérdidas causadas por las plagas han sido un problema importante. Enneothrips flavens ha sido la principal plaga del mani en Brasil. El control de E. flavens se ha realizado utilizando productos químicos sintéticos. El objetivo de este trabajo fue evaluar la patogenicidad de los hongos Beauveria bassiana y Metarhizium anisopliae de productos comerciales y de la colección UNESP / FCAV sobre ninfas de E. flavens. Se aplicaron esporas de estos hongos, a una concentración de $10^{8} \mathrm{~mL}^{-1}$, al folleto de la planta de maní que contenía 10 ninfas de E. flavens. La mortalidad de las ninfas se evaluó después de 3, 5 y 7 días de observación. M. anisopliae (ARSEF 3293) y la cepa de producto comercial (Metarril $\AA$ ) mostraron valores de mortalidad de $34 \%$ y $32 \%$, respectivamente. La cepa B. bassiana del producto comercial (Boverilß) y la cepa B. bassiana (ARSEF 3288) presentaron valores de mortalidad de $76 \%$ y $44 \%$, respectivamente. Los datos obtenidos en este trabajo sugieren que los aislados ARSEF 3293 y ARSEF 3288, así como las cepas comerciales, tienen potencial para ser utilizados como agente de control biológico de E. flavens.
\end{abstract}

Palabras clave: Maní; Control biológico; Trips; Beauveria bassiana; Metarhizium anisopliae.

\section{Introdução}

O amendoim (Arachis hypogaea, L.) faz parte da cultura brasileira e são consumidos principalmente na forma de grãos, torrados ou cozidos. Os frutos do amendoim (Arachis hypogaea, L.) são importante fonte de energia pois são ricos em lipídeos, proteínas e vitaminas (MACÊDO, 2007). No Brasil, na safra 2019/2020, foram produzidas aproximadamente 422.2 mil toneladas de amendoim sendo que 406.50 mil foram produzidas no Estado de São Paulo, o que representa 96\% da produção nacional (CONAB, 2020). A produção de amendoim no Brasil tem aumentado nos últimos anos e o país ocupa atualmente a décima primeira posição mundial. 
O aumento na produção de amendoim no Brasil se deve principalmente ao uso de cultivares mais produtivas, adoção de sistemas de produção tecnificados e a redução dos custos de produção agrícola. Entretanto, fatores tais como clima e ocorrência de pragas têm causado perdas significativas na produção. O tripes-do-prateamento Enneothrips flavens Moulton (Thysanoptera: Thripidae) tem sido descrito como a principal praga do amendoim (Arachis hypogea L.) no Brasil (MONTEIRO; MOUND; ZUCCHI, 2000). O ciclo de vida de E. flavens dura cerca de 13 dias, passando pelos estágios de ovo, dois estágios imaturos (ninfas I e II), dois estágios quiescentes (pré-pupa e pupa) e adultos (MOUND; TEULON, 1995).

Os insetos podem afetar as diferentes partes da planta, tais como folhas, raízes, vagens e sementes (SANTOS; FREIRE; SUASSUMA, 2007). Esta praga causa prejuízo em decorrência do ataque aos folíolos jovens das plantas, que quando se abrem evidenciam estrias e deformações, apresentando aspecto de prateamento (MORAES et al., 2005).

Alguns autores observaram que dependendo da cultivar, a falta de controle de E. flavens reduz a emissão de brotos e o desenvolvimento vegetativo das plantas (LOURENÇÃO et al., 2007). Quando não controlada, essa praga é capaz de reduzir a produtividade em até $62,7 \%$, dependendo a população do inseto, da cultivar utilizada e do local de plantio (MORAES et al., 2005).

O método de controle largamente usado para redução de populações da praga é o químico, aplicado de forma calendarizada a cada 15 dias durante todo o desenvolvimento da cultura (MICHELOTTO et al., 2017). Porém, o comportamento de se alocar no interior de folíolos fechados reduz a eficiência do controle químico, uma vez que não têm contato direto com o produto. O uso de inseticidas sistêmicos tem sido o mais utilizado por ser mais eficiente no controle de pragas. $O$ uso destes defensivos traz problemas de impacto ambiental, custos de produção e principalmente resistência da praga a diferentes inseticidas. 
Esse cenário força a busca por estratégias diversificadas de controle, como a resistência de genótipos de amendoim (BOIÇA JÚNIOR et al., 2012; MICHELOTTO et al., 2017; PIROTTA et al., 2017). Entretanto, alguns autores relatam a dificuldade de obtenção desses genótipos, pois os materiais comerciais diferem em número de cromossomos daqueles silvestres que possuem alta resistência a pragas (ROBLEDO; SEIJO, 2008; 2010).

Outra estratégia importante é a utilização do manejo integrado de pragas através do controle biológico utilizando microrganismos. Dentre os fungos entomopatogênicos, $B$. bassiana e $M$. anisoplae tem sido os mais estudados nas pesquisas e utilizados nas formulações de produtos comerciais (LITWIN; NOWAK, RÓŻALSKA, 2020).

O uso de controle biológico de E. flavens na cultura do amendoim foi relatado apenas com o uso de predadores da família Chrysopidae (RODRIGUES et al., 2014). Neste trabalho os autores observaram que as plantas que receberam ovos embrionados e larvas de primeiro instar de Chrysoperla externa apresentaram redução significativa no número médio de tripes (RODRIGUES et al., 2014).

Entretanto, em outras espécies de tripes já foi relatado o uso de fungos entomopatogênicos como Beauveria bassiana (Balsamo) (LEE et al., 2017) e Metarhizium anisopliae (Mestch.) (LOPES; ALVES; TAMAI, 2000; VESTERGAARD et al.,1995) em culturas de tomate, pepino e alface. Os estudos mostraram que estes fungos foram capazes de causar $90 \%$ de mortalidade de Frankliniella occidentalis, valores semelhantes aos obtidos com o emprego de controle químico.

No Brasil produtos à base destes fungos têm sido utilizado em programas de controle biológico de pragas em diferentes culturas. $O$ desenvolvimento de um programa de controle biológico de pragas depende do isolamento e caracterização de novos isolados de fungos entomopatogênicos e deve ser a etapa inicial. Nesse contexto, objetivou-se com este trabalho avaliar a suscetibilidade de E. flavens aos fungos 
entomopatogênicos B. bassiana e $M$. anisopliae da coleção do Laboratório de Controle Microbiano de Artrópodes Praga da UNESP/FCAV e de dois produtos comerciais.

\section{Material e métodos}

\section{Coleta de Enneothrips flavens}

Ninfas de E. flavens foram obtidas de plantas de amendoim (Arachis hypogea L.) cultivadas em áreas comerciais na região de Jaboticabal, São Paulo, Brasil. Tendo sido constatada infestação de tripes na área, folíolos fechados foram coletados e acondicionados em placas de Petri providas de algodão hidrofílico úmido, objetivando preservar o turgor dos folíolos.

\section{Fungos entomopatógenos}

Foram testados dois produtos comerciais formulados com Beauveria bassiana (Boveri(@) e Metarhizium anisopliae (Metarril( $)$ ), ambos fornecidos pela empresa Koppert Brasil - Produtos Biológicos (Piracicaba, São Paulo, Brasil). Foram também utilizados isolados provenientes do Laboratório de Controle Microbiano de Artrópodes Praga da UNESP/FCAV, Beauveria bassiana (ARSEF 3288) e Metarhizium anisopliae (ARSEF 3293). Para todos os isolados utilizados foi realizado teste de viabilidade, no qual foi obtido valores acima de $70 \%$.

Os isolados usados neste trabalho foram multiplicados em placas de Petri contendo meio BDA (Batata, dextrose, ágar) e incubadas a $26 \pm 1{ }^{\circ} \mathrm{C}$ e 12 horas de fotoperíodo, por sete dias. Após esse tempo, $10 \mathrm{~mL}$ de água destilada esterilizada contendo Tween $\AA 80(0,01 \%)$ foram adicionados nas placas e os conídios coletados por raspagem da superfície do meio de cultura. A suspensão obtida foi diluída em solução salina $(0.9 \%)$ e a quantidade de conídios contadas em uma câmara de Neubauer afim de padronizar a concentração em 1 × $10^{8}$ conídios $/ \mathrm{mL}$. 


\section{Teste de patogenicidade}

Para determinar a patogenicidade indireta de isolados de $M$. anisopliae e B. bassiana a E. flavens quando aplicado sobre o folíolo, foi montado experimento com parcelas compostas por 10 ninfas e cada tratamento replicado 10 vezes. Os tratamentos utilizados são: $(I)$ - isolado $M$. anisopliae (ARSEF 3293) contendo $10^{8}$ conídios/ $\mathrm{mL}+$ adjuvante $(0,05 \%)$; (ii) - produto comercial M. anisopliae (Metarril(囚) contendo $10^{8}$ conídios/ $\mathrm{mL}$ + adjuvante (0,05\%); (iii) - isolado B. bassiana (ARSEF 3288) contendo $10^{8}$ conídios/ $\mathrm{mL}+$ adjuvante (0,05\%); (iv) - produto comercial B. bassiana (Boveril $\Theta$ ) contendo $10^{8}$ conídios/ $\mathrm{mL}+$ adjuvante $(0,05 \%) ;(v)$ - testemunha (água destilada + adjuvante $(0,05 \%)$ ).

As ninfas foram dispostas em folíolos, sendo que cada folíolo abrigará 10 ninfas de E. flavens, sendo em sequência colocado em placas de Petri de $90 \mathrm{~mm}$ de diâmetro sobre papel filtro. Foi aplicado $1 \mathrm{~mL}$ de suspensão de conídios de $M$. anisopliae ou $B$. bassiana com o auxílio da Torre de Potter. Os folíolos infestados foram colocados em placas de Petri de $70 \mathrm{~mm}$ de diâmetro contendo folíolos de amendoim e foram selados com plástico filme para manter a umidade. No tratamento controle as ninfas foram pulverizadas com água destilada e adjuvante (0,05\%) (ADAPTADO DE THUNGRABEAD, 2007).

As placas de Petri foram colocadas em B.O.D. regulada a $26 \pm 2^{\circ} \mathrm{C}$, $60-70 \%$ U. R. e fotoperíodo de 13L:11E (GAO, 2012).

As avaliações de mortalidade confirmada e observada foram realizadas após 3, 5 e 7 dias após a aplicação dos tratamentos, pela observação de esporulação na camada externa do inseto. Para essa avaliação os folíolos foram abertos com o uso de pinças de pontas finas.

\section{Análise estatística}

As comparações entre as medias foram feitas pelo teste de Tukey a $5 \%$ de probabilidade, por meio do programa estatístico Statistical Analysis Sistem (SAS) (SAS, 2015). 


\section{Resultados e discussão}

Durante o ciclo fenológico da cultura do amendoim diversos insetos e ácaros podem ocorrer, atacando tanto raízes quanto a parte aérea das plantas. As principais pragas de parte aérea são causadas por E. flavens, Caliothrips brasiliensis e Frankiliniella shultzei (DALVA, 2016). Neste trabalho foi comparado a virulência de $B$. bassiana (ARSEF 3288) e $M$. anisoplae (ARSEF 3293), da coleção da UNESP/FCAV, com dois produtos comercializados no Brasil a base destes fungos.

Os isolados dos fungos $M$. anisopliae e $B$. bassianaforam capazes de colonizar as ninfas de $E$. flavens. No levantamento inicial, realizado após 3 dias de aplicação da suspensão de conídios dos isolados $\left(10^{8}\right.$ conídios $\mathrm{mL}^{-}$ 1) observamos uma mortalidade significativa das ninfas de $E$. flavens, principalmente nos tratamentos com o produto comercial Boveril. $\mathrm{O}$ isolado comercial e o ARSEF 3293 de M. anisopliae provocaram uma morte de 24 e $16 \%$ das ninfas, respectivamente (Figura $1 \mathrm{~A}$ ). $\mathrm{O}$ isolado comercial e $\mathrm{O}$ ARSEF 3288 de B. bassiana provocaram uma morte de 38 e $20 \%$, respectivamente. Enquanto na testemunha houve uma morte de $4 \%$ das ninfas E. flavens.

Após 5 dias de aplicação o isolado comercial e o ARSEF 3293 de $M$. anisopliae provocaram uma morte de 30 e $28 \%$ das ninfas, respectivamente (Figura 1B). O isolado comercial e o ARSEF 3288 de $B$. bassiana provocaram uma morte de 40 e $24 \%$, respectivamente. Enquanto na testemunha houve uma morte de $10 \%$ das ninfas E. flavens.

No levantamento final, realizado após 7 dias de aplicação da suspensão de esporos o isolado comercial e o ARSEF 3293 de M. anisopliae provocaram uma morte de 34 e $32 \%$ das ninfas, respectivamente (Figura 1C). $O$ isolado comercial e o ARSEF 3288 de $B$. bassiana provocaram uma morte de 76 e $44 \%$, respectivamente (Figura 1C). Enquanto na testemunha houve uma morte de $12 \%$ das ninfas E. flavens (Figura 1C). De modo geral o isolado de $B$. bassiana do produto comercial Boveril apresentou as maiores médias, quando comparadas aos outros tratamentos (Figura 1). 
Os resultados obtidos mostraram que $\mathrm{O}$ isolado de $M$. anisopliae (ARSEF 3293 da coleção da UNESP/FCAV foi eficiente na mortalidade das ninfas de $E$. flavens com valores comparados com aqueles obtidos com o produto comercial (Metarril $($ )). Entretanto, $\mathrm{O}$ isolado de $B$. bassiana do produto comercial (Boveril( $\Theta$ ) foi mais eficiente na mortalidade das ninfas de E. flavens, quando comparado com o isolado de B. bassiana (ARSEF 3288). Em geral isolados de $B$. bassiana foram mais virulentos do que os isolados de M. anisopliae.

Resultados semelhantes foram encontrados por DHYEGO et al. (2014) que avaliaram $O$ potencial de 49 isolados dos fungos entomopatogênicos das espécies $B$. bassiana e $M$. anisopliae, contra lagartas de Spodoptera frugiperda. Os autores concluíram que os isolados de $B$. bassiana apresentaram maior patogenicidade contra lagartas de $S$. frugiperda quando comparado com isolados de M. anisopliae.

Os produtos comerciais que apresentam conídios dos fungos $B$. bassiana e $M$. anisoplae são muito utilizados e eficientes para o controle biológico de diversas pragas (LITWIN et al., 2020). Seus conídios podem penetrar na cutícula do inseto e são mediados pela ação de enzimas hidrolíticas (proteases e quitinases) e metabolitos secundários. Depois de atravessar a cutícula dos hospedeiros, formam-se tubos germinativos e hifas que atravessam o tegumento e chegam até a hemolinfa matando o inseto (LITWIN et al., 2020). 

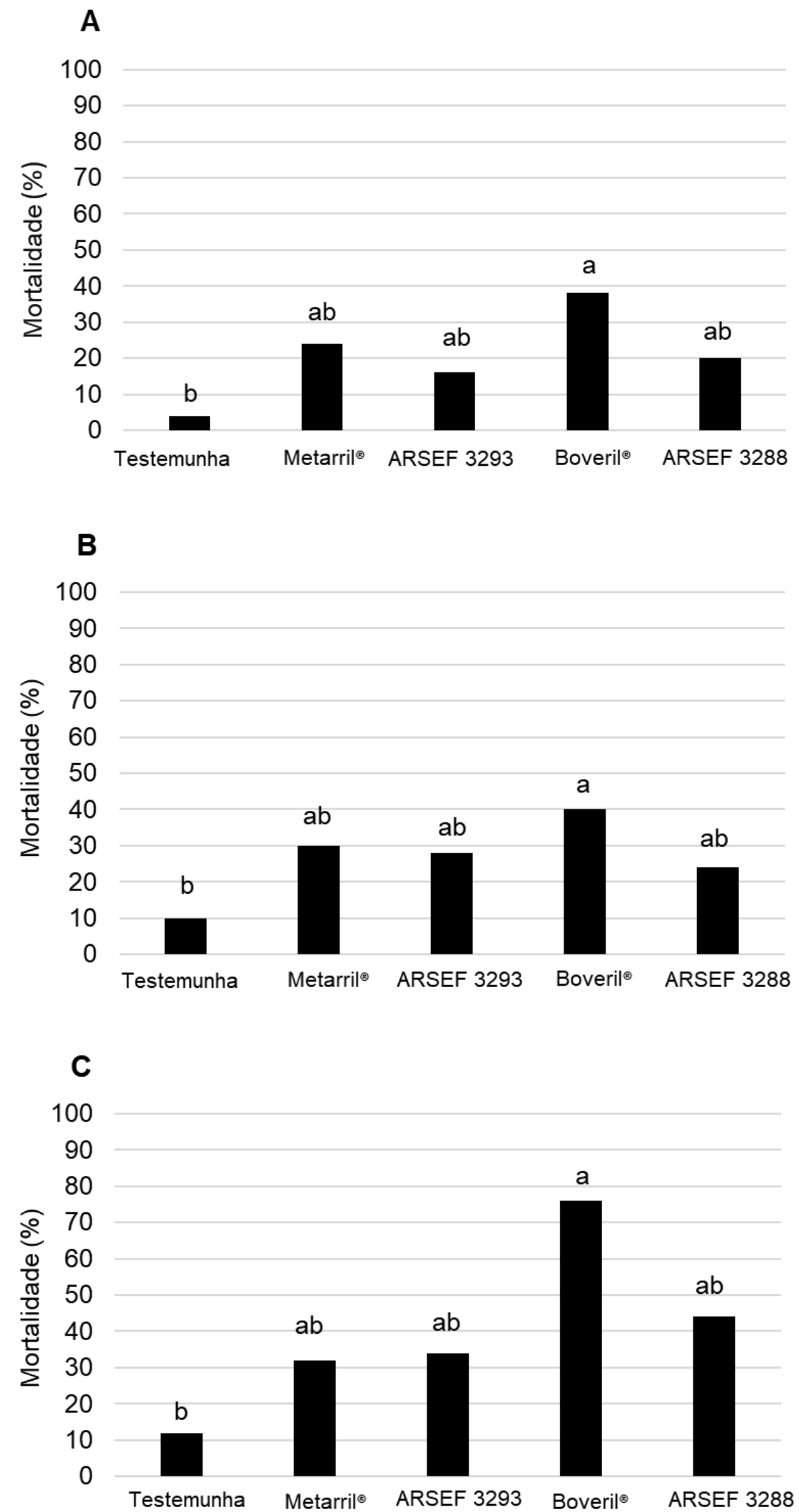

Figura 1. Mortalidade (\%) de ninfas de Enneothrips flavens com 3 dias (A), 5 dias (B) e 7 dias (C) após a aplicação dos fungos entomopatogênicos. Médias com a mesma letra não diferem entre si, pelo teste de Tukey, a 5\% de probabilidade. 


\section{Conclusões}

Os resultados obtidos neste trabalho mostram que os isolados $M$. anisopliae (ARSEF 3293) e B. bassiana (ARSEF 3288) possuem potencial para serem utilizados como agentes de controle biológico de $E$. flavens. Entretanto, outros trabalhos precisam ser feitos para avaliar os mecanismos de ação destes isolados contra E. flavens, custo de produção em escala comercial, vida útil dos esporos, desenvolvimento de formulações e tempo de prateleira para que possam ser utilizados como produto comercial.

\section{Agradecimentos}

À CAPES (Coordenação de Aperfeiçoamento de Pessoal de Nível Superior), ao CNPq (Conselho Nacional de Desenvolvimento Científico e Tecnológico) pelo apoio a esta pesquisa e a empresa Koppert pelo fornecimento dos produtos comerciais. 


\section{Referências}

BOIÇA-JÚNIOR, Arlindo Leal et al. Avaliação de resistência de cultivares de amendoim de hábito de crescimento rasteiro a Enneothrips flavens Moulton (Thysanoptera: Thripidae). Arquivos do Instituto Biológico, São Paulo, v. 79, n. 1, p. 33-38, 2012.

CONAB. Acompanhamento da safra brasileira de grãos, V. 7 - Safra 2019/20 - Décimo levantamento, Brasília, p. 1-31. julho 2020.

DALVA, Gabriel. Pragas do amendoim. Arquivos do Instituto Biológico, São Paulo, V. 26, p. 1-25, 2016.

DHYEGO, Thomazoni; FORMENTINI, Marina Andressa; ALVES, Luis Francisco Angeli. Patogenicity of entomopathogenic fungi to Spodoptera frugiperda (Smith) (Lepidoptera: Noctuidae). Arquivos do Instituto de Biologia, [s.I.], v. 81 (2), p. 126-133, 2014

LEE, Se Jin et al. Entomopathogenic Beauveria bassiana granules to control soil-dwelling stage of western flower thrips, Frankliniella occidentalis (Thysanoptera: Thripidae). Biocontrol, [s.I.], v. 62 n, 5, p. 639-648, 2017.

LITWIN, Anna; NOWAK, Monika; RÓŻALSKA, Sylwia. Entomopathogenic fungi: unconventional applications. Reviews in Environmental Science and Biotechnology, [s.I.], v. 19, 23-42, 2020.

LOPES, Rogério Biaggioni; ALVES, Sérgio Batista; TAMAI, Marco Antonio. Fungo Metarhizium anisopliae e o controle de Frankliniella occidentalis em alface hidropônico. Scientia Agricola. [online], v. 57 (2), p. 239-243, 2000.

LOURENÇÃO, André Luiz et al. Fitossanidade efeito da infestação de Enneothrips flavens moulton sobre o desenvolvimento de cultivares de amendoim. Bragantia, Campinas, v.66, n.4, p.623-636, 2007.

MICHELOTTO, Marcos Doniseti et al. Resistance to thrips (Enneothrips flavens) in wild and amphidiploid Arachis species. Plos One, [s.I.], v. 12, n. 5, p. e0176811, 2017.

MONTEIRO, Renata; MOUND, Laurence; ZUCCHI, Roberto Antônio. Thrips (Thysanoptera) as pests of plant production in Brazil. Revista Brasileira de Entomologia, [s.I.], v. 43, p.163-171, 2000.

MORAES, Andrea Rocha Almeida et al. Infestation by Enneothrips flavens Moulton and yield of peanut cultivars. Scientia Agricola, [s.I.], v. 62, n. 5, p. 469-472, 2005.

MOUND, Laurence; TEULON, David Austin John. Thysanoptera as phytophagous opportunists. In: PARKER, B. L.; SKINNER, M; LEWIS, T. (Ed.). Thrips biology and management. New York: Plenum Publishing Corporation, 1995. p.3-20. 
PIROTTA, Maria Zacarelli et al. Resistance to Enneothrips flavens Moulton and genetic parameters estimation in interspecific genotypes of peanut. Acta Scientiarum. Agronomy, [s.I.], v. 39, n. 3, p. 339-348, 2017.

ROBLEDO, Germán; SEIJO, Guillermo. Characterization of the Arachis (Leguminosae) D genome using fluorescence in situ hybridization (FISH) chromosome markers and total genome DNA hybridization. Genetics and Molecular Biology, [s.I.], v. 31, n.3, p. 717-724, 2008.

ROBLEDO, Germán; SEIJO, Guillermo. Species relationships among the wild $\mathrm{B}$ genome of Arachis species (section Arachis) based on FISH mapping of rDNA loci and heterochromatin detection: a new proposal for genome arrangement. Theoretical and Applied Genetics, [s.I.], v. 121, n. 6, p. 10331046, 2010.

RODRIGUES, Camilla Alves et al. Dynamics and predation efficiency of Chrysoperla externa (Neuroptera: Chrysopidae) on Enneothrips flavens (Thysanoptera: Thripidae). Florida Entomologist, [s.l.], v. 97, n. 2, p. 653658, 2014.

SANTOS, Roseane Calvacanti; FREIRE, Rosa Maria Mendes; SUASSUNA, Tais de Moraes Falleiro. Amendoim : o produtor pergunta, a Embrapa responde / editores técnicos, - Brasília, DF : Embrapa Informação Tecnológica, 2009. 240 p. il. ; $22 \mathrm{~cm}$ - (Coleção 500 perguntas, 500 respostas).

SAS Institute (2015) SAS/IML 14.1 User's Guide. Cary, NC: SAS Institute Inc

VESTERGAARD, Susanne et al. Pathogenicity of the hyphomycete fungi Verticillium lecanii and Metarhizium anisopliae to the western flower thrips, Frankliniella occidentalis. Biocontrol Science and Technology, [s.I.], v.5, p. 185-192, 1995. 